\title{
The motion of the soil freezing front with account of the temperature distribution
}

\author{
Eduard Arinshtein ${ }^{1}$, Aleksey Tatosov ${ }^{1}$, Vladimir Kutrunov ${ }^{1}$, and Yulia Yanbikova ${ }^{1, *}$ \\ ${ }^{1}$ University of Tyumen, Volodarskogo Street, 6, Tyumen, 625003, Russia
}

\begin{abstract}
The Stefan problem in the heat propagation in a two-phase region, which is relevant in the study of the processes of motion of the freezing boundary, taking into account the properties of both frozen and thawed soil, has been considered. In contrast to the standard approach to solving the Stefan problem, when the temperature of one phase is fixed, the paper considers the case when the heat fluxes are determined by the temperature distribution both in one and the other phase. This allows us to clarify the process of the phase boundary motion (the boundary of soil freezing), which must be taken into account when founding the carcasses of the construction of high-rise buildings. The solution is given in a form that makes it possible to implement by numerical methods on an accessible electronic computer technology.
\end{abstract}

\section{Introduction}

The study of soil freeze and thaw processes, the boundary movement between these phases is very important for the successful solution of many problems arising in the development of the northern territories [1-6].

The problem of the front motion of the phase transition, the Stefan problem, comes to solving the heat conduction equation $[7,8]$ in a region with a moving boundary which velocity of motion is limited by the transfer (or withdrawal) of the transition heat absorbed (or released) during this transition. It is usually assumed that the temperature is different from the transition temperature only in the region occupied by a new phase, whereas the medium has a temperature equal to the phase transition temperature [9]. Obviously, the speed of the front (the boundary of a new phase) is affected by the heat transfer not only in the region of a new phase, but also in the medium in the outer region occupied by the initial phase. The heat transfer in the new phase is determined by the temperature at the boundary from which the growth of the new phase begins, the heat transfer in the initial phase is determined by the initial temperature of the medium. If the temperature is measured from the temperature of the phase transition, the boundary and initial temperatures must have different signs. For definiteness, we assume that the boundary temperature is higher and the initial temperature is lower than the transition temperature, which corresponds to the melting process. To describe the crystallization process, it is sufficient to change the signs of the boundary and initial conditions and the temperature in each region.

\footnotetext{
* Corresponding author: y.f.yanbikova@utmn.ru
} 
At the first stage, we consider the one-dimensional problem of the temperature distribution in the region $x \geq 0, t \geq 0$, satisfying the heat equation

$$
\frac{\partial u}{\partial t}-\frac{\partial u^{2}}{\partial x^{2}}=0
$$

with boundary condition $u(0, t)=f(t)$ and the initial condition $u(x, 0)=-g(x)(f(t)>0$ и $g(x)>0)$. The Green's function of this problem is known [10], it is equal to

$$
G(x, t ; \xi, \tau)=\frac{1}{2 \sqrt{\pi(t-\tau)}}\left(e^{-\frac{(x-\xi)^{2}}{4(t-\tau)}}-e^{-\frac{(x+\xi)^{2}}{4(t-\tau)}}\right)
$$

which makes it possible to obtain a solution in a closed form:

$$
\begin{gathered}
u(x, t)=\int_{0}^{t} f(\tau) e^{-\frac{x^{2}}{4(t-\tau)}} \frac{x d \tau}{2 \sqrt{\pi}(t-\tau)^{3 / 2}}-\int_{0}^{\infty} g(\xi)\left(e^{-\frac{(x-\xi)^{2}}{4 t}}-e^{\left.-\frac{(x+\xi)^{2}}{4 t}\right)} \frac{d \zeta}{2 \sqrt{\pi t}}=\right. \\
=\int_{\frac{x}{2 \sqrt{t}}}^{\infty} f\left(t-\frac{x^{2}}{4 z^{2}}\right) e^{-z^{2} \frac{d z}{\sqrt{\pi}}-\int_{-\frac{x}{2 \sqrt{t}}}^{\infty} g(2 z \sqrt{t}+x) e^{-z^{2}} \frac{d z}{\sqrt{\pi}}+\int_{\frac{x}{2 \sqrt{t}}}^{\infty} g(2 z \sqrt{t}-x) e^{-z^{2}} \frac{d z}{\sqrt{\pi}}}
\end{gathered}
$$

For sufficiently large times, when the deviations of the functions $f(t)$ and $g(x)$ from the mean values can be neglected, the expression (3) yields the asymptotic solution

$$
u(x, t)=\frac{f}{\sqrt{\pi}} \int_{Z_{0}}^{\infty} e^{-z^{2}} d z-\frac{2 g}{\sqrt{\pi}} \int_{0}^{Z_{0}} e^{-z^{2}} d z=f\left(1-\Phi\left(Z_{0}\right)\right)-2 g \Phi\left(Z_{0}\right)
$$

where $Z_{0}=\frac{x}{2 \sqrt{t}}, \Phi\left(Z_{0}\right)=\frac{1}{\sqrt{\pi}} \int_{0}^{Z_{0}} e^{-z^{2}} d z$ is the probability integral.

The mean values of $f$ and $g$ functions $f(t)$ and $g(x)$ are constants. The condition $u(x, t)=0$ determines the displacement of the front $l(t)=2 a \sqrt{t}$ of the phase transition temperature, where the constant $a$ is the only solution of equation

$$
f\left(1-\Phi\left(Z_{0}\right)\right)=2 g \Phi\left(Z_{0}\right)
$$

which exists for any non-negative values of $f$ and $g$.

The temperature gradient that determines the heat flux is equal on this front

$$
-\frac{\partial u}{\partial x}=\frac{f+2 g}{2 \sqrt{\pi t}} e^{-a^{2}}
$$

In the case when the absorption (or separation) of the transition heat is small, for example, 
in processes in a dense ground with a low concentration of moisture, the solution obtained is an approximate solution of the Stefan problem. On its basis, it is possible to obtain a solution of the Stefan problem in other cases.

\section{Temperature distribution in the layer of the new phase}

The expression (4) defines the solution for $0 \leq x \leq l(t)$ and the boundary conditions $u(0, t)=f, u(l(t), t)=0$. The continuation of the solution for $x \geq l(t)$ is purely formal, the parameter $g=g_{1}$ is an auxiliary quantity, which can be expressed in terms of the parameter $a$ from the equation (5). The parameter $a$ is determined by the heat transfer conditions for $x=l(t)$. In a particular case, if in the external environment the temperature is equal to the transition temperature and, therefore, is constant, the temperature gradient and the heat flux for $x \geq l(t)$ are zero. The condition that the heat flux in the layer is equal to the rate of absorption (or separation) of the transition heat and, consequently, is proportional to the velocity of the front of the phase transition, determines the parameter $a$ :

$$
-\frac{\partial u}{\partial x}=\frac{f+2 g_{1}}{2 \sqrt{\pi t}} e^{-a^{2}}=\lambda \frac{d l}{d t}=\lambda \frac{a}{\sqrt{t}}
$$

Eliminating the auxiliary quantity $g_{1}$, from equations (7) and (5) (replacing $g \rightarrow g_{1}$ in Eq. (5)), we obtain a condition that uniquely determines the parameter $a$ and the motion of the phase transition front.

\section{Temperature distribution in the external environment}

The expression (4) also defines the temperature distribution for $x \geq l(t)$ under the boundary condition $u(l(t), t)=0$ and the initial condition $u(x, 0)=-g$. The thermal diffusivity of the external medium may differ from the thermal diffusivity of the new phase. This difference can be taken into account by replacing $t \rightarrow \gamma^{2} t$ and $a \rightarrow a / \gamma$, where $\gamma^{2}$ is the ratio of thermal diffusivity. The continuation of the solution for $x \leq l(t)$ is purely formal and, using the equation (5), determines the auxiliary quantity $f_{1}=f / \gamma^{2}$. The heat flux in the vicinity of the front is

$$
\gamma^{2} \frac{\partial u}{\partial x}=\frac{\left(f_{1}+2 g\right) \sqrt{\gamma}}{2 \sqrt{\pi t}} e^{-\frac{a^{2}}{\gamma^{2}}}
$$

This heat flux determines not the influx of heat from the melt, but the outflow of heat from the fusion front to the colder solid phase.

The value of the parameter $a$ is determined by the condition of the heat balance, which now takes the form:

$$
-\left.\frac{\partial u}{\partial x}\right|_{x=l-0}+\left.\gamma \frac{\partial u}{\partial x}\right|_{x=l+0}=\lambda \frac{d l}{d t}=\lambda \frac{a}{\sqrt{t}}
$$


The equation (5) for $x=l-0$ and for $x=l+0$ gives

$$
2 g_{1}=f \frac{1-\Phi(a)}{\Phi(a)}, f+2 g_{1}=\frac{f}{\Phi(a)}, f_{1}=2 g \frac{\Phi(a / \gamma)}{1-\Phi(a / \gamma)}, f_{1}+2 g=\frac{2 g}{1-\Phi(a / \gamma)},
$$

which leads to the equation

$$
f-\frac{2 g \Phi(a / \gamma) e^{\frac{\left(\gamma^{2}-1\right) a^{2}}{\gamma^{2}}}}{1-\Phi(a)}=2 a e^{a^{2}} \sqrt{\pi} \Phi(a),
$$

which determines the parameter $a$ and, hence, the velocity of the phase transition front. The right-hand side of the equation (11) is a monotonic function of the parameter $a$ rapidly increasing from zero, the function $\Phi(a / \gamma) \rightarrow 0$ as $a \rightarrow 0$, the left side of the equation when the parameter $a$ increases from zero decreases from the positive value of $f$, therefore the equation (11) has the only solution for any values of the parameters $f$ and $g$. As the parameter $g$ grows, the value of the parameter $a$, which is a solution of the equation (11), decreases.

It follows that the low temperature outside the melting front slows down, but does not stop the movement of the front.

\section{Conclusions}

Forecasting the annual temperature fluctuations and the possible movements of the melt zone boundary is necessary when designing the construction of industrial and residential structures in the Far North. This requires a solution of the Stefan problem in a form that can be used by a design engineer. The solution obtained satisfies this condition. For a more accurate (quantitative) description of the properties of the solution obtained, it is sufficient to use any known programming method and computer technology, which is available in the vast majority of design organizations.

\section{References}

1. E.D. Yershov, Y.P. Akimov, V.G. Cheverev, E.Z. Kuchukov, Fazovyj sostav vlagi v merzlyh porodah (Moscow State University Publishing House, 1979)

2. Y.B. Gorelik, V.S. Kolunin, Fizika $i$ modelirovanie kriogennyh processov v litosfere (GEO, 2002)

3. I.I. Nesterov, Y.S. Daniehlyan, P.A. Yanickij, Doklady AN SSSR 287(5), 1127-1130 (1986)

4. I.I. Nesterov, Y.S. Daniehlyan, P.A. Yanickij, Doklady AN SSSR 277(4), 928-932 (1984)

5. R.I. Medvedskij, Stroitel'stvo i ehkspluataciya skvazhin na neft' i gaz v vechnomerzlyh porodah (Nedra, 1987)

6. V.T. Sedov, Teploobmen pri burenii merzlyh porod (Nedra, 1990)

7. A.V. Lykov, Teoriya teploprovodnosti (Vysshaya shkola, 1997)

8. G. Karslou, D. Eger, Teploprovodnost' tverdyh tel (Nauka, 1994)

9. G.M. Dolgih, G.V. Anikin, I.P. Rilo, et. al. Earth cryosphere XIX(1) (2015)

10. F. Trikomi, Differencial'nye uravneniya (Izdatel'stvo inostrannoy literatury, 1962) 